\title{
Les travailleurs des plateformes juridiques en France : le juge, arbitre de leur statut?
}

Isabelle Desbarats

\section{(2) OpenEdition}

1 Journals

Édition électronique

URL : https://journals.openedition.org/rdctss/1551

DOI : 10.4000/rdctss. 1551

ISSN : 2262-9815

Éditeur

Centre de droit comparé du travail et de la sécurité sociale

Édition imprimée

Date de publication : 1 juin 2019

Pagination : 24-31

ISSN : 2117-4350

\section{Référence électronique}

Isabelle Desbarats, «Les travailleurs des plateformes juridiques en France : le juge, arbitre de leur statut ? », Revue de droit comparé du travail et de la sécurité sociale [En ligne], 2 | 2019, mis en ligne le 01 novembre 2021, consulté le 11 novembre 2021. URL : http://journals.openedition.org/rdctss/1551 ; DOI : https://doi.org/10.4000/rdctss. 1551

\section{cc)}

Revue de droit comparé du travail et de la sécurité sociale est mise à disposition selon les termes de la Licence Creative Commons Attribution - Pas d'Utilisation Commerciale - Pas de Modification 4.0 International. 


\section{LES TRAVAILLEURS DES PLATEFORMES JURIDIQUES EN FRANCE : LE JUGE, ARBITRE DE LEUR STATUT ?}

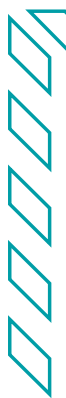

\section{ISABELLE DESBARATS}

Professeure, Centre de Droit des Affaires, Université Toulouse I Capitole

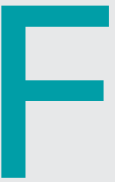

aut-il ranger le travailleur 2.0 dans le champ des salariés ou des indépendants ?'. S'il est une "vieille question neuve ", c'est bien celle-ci qui renvoie à des problématiques anciennes mais dont les enjeux sont renouvelés à l'ère des plateformes numériques, définies, par l'article L. 111-7I du Code de la consommation, comme «toute personne physique ou morale proposant, à titre professionnel, de manière rémunérée ou non, un service de communication au public reposant sur (...) la mise en relation de plusieurs parties en vue de la vente d'un bien, de la fourniture d'un service ou de l'échange ou du partage d'un contenu, d'un bien ou d'un service ". Vieux débats en effet, qui sont ceux des frontières du droit du travail et que les juges ont tranchés en faisant du critère de la subordination juridique celui permettant de distinguer ce qui relève du salariat de ce qui lui est étranger. Mais nouveaux enjeux aussi, qui sont ceux générés par l'expansion des plateformes numériques dont le succès se fonde justement sur l'éviction de ce droit, via le recours à l'entreprenariat ${ }^{2}$.

Dans ce contexte et alors que les contentieux intentés contre les plateformes se multiplient à l'initiative de certains travailleurs mais aussi d'organismes de protection sociale, c'est une place particulière qu'il convient d'octroyer à la décision que vient de rendre, en France, la Chambre sociale de la Cour de cassation ${ }^{3}$. D'abord, cette décision est la première de cette Cour concernant la nature des rapports contractuels noués entre travailleurs et plateformes numériques, alors que, jusque-là, seules les juridictions du fond se sont prononcées de surcroît en

1 L. Gamet, «Le livreur à vélo, la plateforme et le droit du travail », AJ Contrat, 2019, p.46.

2 M. Julien et E. Mazuyer, "Le droit du travail à l'épreuve des plateformes numériques », Revue de Droit du Travail, 2018, p.189.

3 Droit Social, 28 novembre 2018, n¹7-20.079, à paraître au Bulletin. 
ordre dispersé 4 : une incertitude que la Cour suprême vient donc de lever en se prononçant en faveur de l'existence d'une relation salariée. Ensuite, cette intervention est d'autant plus marquante qu'elle a pour effet d'ériger le juge en une sorte de garant des droits des travailleurs numériques, et donc en rempart de ceux-ci (I). Cependant, la portée de cette solution ne risque-t-elle pas d'être limitée et le rôle protecteur du juge contourné par l'intervention du législateur ?5 (II). Au cœur de ces questions : la fonction d'arbitre du juge.

\section{I - AUJOURD'HUI - LE JUGE, GARANT DES DROITS DES TRAVAILLEURS NUMÉRIQUES}

Alors que l'économie des plateformes numériques s'appuie sur le statut d'autoentrepreneur des travailleurs ayant recours à leurs services, c'est une réponse fondée sur I'article L 8221-6-I du Code du travail qui devrait être a priori apportée à la question de savoir à quelles conditions les requalifier en salariés. En effet, ces travailleurs étant présumés ne pas être liés avec leur donneur d'ordre par un contrat de travail dans l'exécution de l'activité ayant donné lieu à immatriculation ou inscription, c'est à la condition d'établir un lien de "subordination juridique permanente » à l'égard de ce donneur d'ordre qu'une telle requalification devrait être subordonnée : une opération d'autant plus utile pour le travailleur, lorsque, comme c'était le cas en l'espèce, la plateforme est mise en liquidation judiciaire. Or il s'avère que si la Chambre sociale a focalisé son attention sur la réalité d'un lien de subordination juridique, l'exigence de permanence n'a pas été évoquée. II en ressort une solution qui, d'un côté, paraît " de facture classique " ${ }^{6}$ tout en présentant un aspect novateur (A), et d'un autre, semble rigoureuse tout en étant pragmatique (B) : au final, une solution nuancée, mais dont l'impact dans le champ de l'économie collaborative ne saurait être minoré7, au point d'être qualifiée de « mauvais coup » par l'Observatoire du travail indépendant ${ }^{8}$.

\section{A - De La (RE)QualificATION DES RELATIONS NOUÉES AVEC UNE PLATEForme NUMÉRIQUE : UNE ANALYSE CLASSIQUE AUX ACCENTS MODERNISTES}

De quoi s'agissait-il ? Un travailleur inscrit en tant qu'autoentrepreneur conclut un contrat de prestation de services avec la société Take Eat Easy afin d'effectuer des livraisons de repas à domicile moyennant une rémunération. Pour réaliser son activité, cette société utilisait une plateforme et une application afin de mettre en relation des restaurateurs partenaires, des clients passant commande de repas par le biais de cette plateforme et des livreurs à vélo. Victime d'accidents de la circulation, un livreur intente une action devant le Conseil de prud'hommes de Paris en demande de requalification de son contrat en un contrat de travail, mais est débouté par les juges du fond. Entretemps, la liquidation judiciaire de la société Take Eat Easy est prononcée mais le mandataire liquidateur refuse

4 A. Fabre, "Les travailleurs des plateformes sont-ils des salariés ? Premières réponses frileuses des juges français », Droit Social, 2018, p. 547.

5 L. Bento de Carvalho et S. Tourneaux, «Actualité du régime juridique du contrat de travail », Droit Social, 2019, p.57.

6 J-Y. Huglo, «Take Eat Easy : une application classique du lien de subordination», Semaine Sociale Lamy, 24 décembre 2018, n 1842-1843.

7 V. Roche, «Travailleurs indépendants et plateformes numériques : I'union impossible ? Le cas de Take Eat Easy », JCP, G, 21 Janvier 2019, n³ 3, p.46.

8 Liaisons sociales Quotidien - L'actualité, 3 décembre 2018, nº 17705. 
d'inscrire au passif de la liquidation les demandes du livreur en paiement des courses effectuées, au motif que celui-ci n'avait jamais été salarié de la société?

Alors qu'il appartenait donc à la Cour de cassation de conclure ou pas à la réalité d'une relation subordonnée entre le livreur et la plateforme, c'est une approche à la fois classique et moderniste qu'elle retient. Classique puisque rappelant, au visa de l'article L. 8221-6-2 du Code du travail, les deux principes gouvernant la qualification de contrat de travail : "l'existence d'une relation de travail ne dépend ni de la volonté exprimée par les parties ni de la dénomination qu'elles ont donnée à leur convention mais des conditions de fait dans lesquelles est exercée l'activité des travailleurs $»^{10} ;$ " le lien de subordination est caractérisé par l'exécution d'un travail sous l'autorité d'un employeur qui a le pouvoir de donner des ordres et des directives, d'en contrôler l'exécution et de sanctionner les manquements de son subordonné $»^{11}(1)$. Mais novatrice également, puisqu'octroyant un rôle notable à ce qui constitue la clé de voûte du travail ubérisé : la géolocalisation (2).

1) «Pas d'attendu révolutionnaire, pas de nouveau principe, un texte bien connu cité au visa... L'arrêt du 28 novembre 2018 ne présente pas de grande originalité du point de vue du droit du travail $»^{12}$... Indéniablement, "la décision est d'une grande sobriété [puisque] la Chambre sociale se contente de réitérer le principe de réalité et les critères classiques de la subordination, [sans s'aventurer] sur d'autres terrains moins stables $»^{13}$, singulièrement celui de la participation du salarié à un service organisé : un choix approuvé par certains ${ }^{14}$, injustifié pour d'autres ${ }^{15}$. Rappelons ici que, « si l'appréciation des éléments de fait permettant de déterminer l'existence ou l'absence d'un lien de subordination relève du pouvoir souverain des juges du fond [...], la Cour de cassation exerce néanmoins un contrôle de motivation pour s'assurer qu'ils tirent les conséquences légales de leur constatation... ${ }^{16}$ : or, c'est justement pour ne pas avoir tiré les conséquences des faits relevés que la Cour suprême casse, pour violation de la loi, la décision litigieuse, ces faits étant selon elle révélateurs de l'existence d'un tel lien, contrairement aux juges du fond.

C'est ainsi d'abord, que, si pour la Cour d'appel, la raison fondamentale s'opposant à une requalification du livreur en salarié tenait au fait qu'il pouvait choisir ses jours et ses horaires de travail ce qui impliquait une «liberté totale " exclusive de toute subordination, cet argument est rejeté par la Cour de cassation suivant en cela l'avocat général, selon lequel une telle liberté ne constitue qu'un indice parmi d'autres de l'absence de lien de subordination ${ }^{17}$. On note ensuite, qu'allant plus loin, la Cour de cassation «mentionne deux éléments qui, selon elle, auraient dû conduire la Cour d'appel à constater l'existence d'un [tel] lien, et donc à requalifier le contrat de prestation de services en contrat de travail $»^{18}$.

9 Voir note explicative de l'arrêt du 28 novembre 2018, op.cit.

10 «Labbanne », Soc., 19 déc. 2000, n²98-40.572, Bull. civ. V, n 437.

11 "Société générale », Soc., 13 nov. 1996, n 94-13.187, Bull. civ. V, n³86.

12 Voir V. Roche, op.cit., et J-Ph. Lhernould, "Les plateformes électroniques de mise en relation rattrapées par le salariat », Jurisprudence Sociale Lamy, 28 janvier 2019, n 468.

13 L. Bento de Carvalho et S. Tourneaux, op.cit.

14 J-Y. Huglo, op.cit.

15 L. Bento de Carvalho et S. Tourneaux, op.cit.

16 B. Bossu, «Plateforme numérique : le droit du travail fait de la résistance », JCP, E, 2019, $\mathrm{n}^{\circ} 3,1031$.

17 Avis C. Courcol-Bouchard, Cass. Soc., 28 nov. 2018, n 17-20.079, JCP S, 2018, 1398.

18 V. Roche, op.cit. 
Le premier tient à la réalité d'un pouvoir de sanction de la plateforme, attestée par un recours possible à plusieurs pénalités graduées, susceptibles de frapper le livreur défaillant et pouvant aller jusqu'à son déréférencement: un système qui, à juste titre selon certains commentateurs $^{19}$, avait été jugé peu significatif par les juges du fond. Quant au second paramètre, il tient à l'existence d'un « système de géolocalisation permettant le suivi en temps réel par la société de la position du coursier et la comptabilisation du nombre total de kilomètres parcourus par celui-ci »: un élément constitutif de «l'ADN » ${ }^{20}$ des plateformes numériques, et dont le recours « rajeunit » une solution qui, à défaut, serait banale.

2) Explicité dans sa note explicative, c'est un rôle important que la Chambre sociale attribue en effet au dispositif de géolocalisation dès lors que les modalités de son recours révélaient que le rôle du donneur d'ordre ne se réduisait pas à une simple activité d'intermédiation ${ }^{21}$. Bien au contraire, elles témoignaient d'un "véritable pouvoir de contrôle des courses, tandis que le système de sanctions [illustrait] le pouvoir disciplinaire de l'employeur ${ }^{22}$ : une analyse cependant peu convaincante pour certains, au motif qu'on ne voit pas « en quoi géolocaliser le livreur, pour calculer sa rémunération en fonction du nombre de kilomètres parcourus, [constituerait] un élément caractérisant la subordination $»^{23}$, d'autres regrettant, pour leur part, que « le pouvoir de donner des ordres et des directives » soit « moins clairement établi » ${ }^{24}$.

Au regard de la Cour de cassation, la conclusion s'imposait cependant. En refusant de conclure à l'existence d'une relation subordonnée, la Cour d'appel n'avait pas tiré toutes les conséquences légales de ses constatations dont l'existence d'un pouvoir de direction et de contrôle pouvait pourtant être déduite. Pour autant, la requalification opérée par la Cour de cassation ne saurait être réduite à la seule application des critères de la subordination juridique encore qu'ajustés aux particularismes des plateformes numériques : elle repose également sur un raisonnement rigoureux - parce que n'évoquant pas l'exigence de «permanence »-, qui est cependant empreint de pragmatisme.

\section{B - De la substitution du STATUt de SALARIÉ À CELUI d'AutoentrepREneUR : UNE JUSTIFICATION RIGOUREUSE MAIS PRAGMATIQUE}

Si elle contredit le raisonnement jusque-là généralement retenu par les juridictions du fond ${ }^{25}$, tant s'en faut que la solution retenue dans l'arrêt Take Eat Easy ait vocation à s'appliquer à toutes les plateformes : «une [...] généralisation [qui contredirait le] principe de réalité selon lequel la [requalification] repose sur les conditions de fait dans lesquelles l'activité est exercée ${ }^{26}$. Conséquence ? Chaque plateforme devrait " être amenée à s'interroger sur le bien-fondé de son modèle à partir des lignes directrices formulées par

19 L. Gamet, op.cit.

20 F. Champeaux, "Travailleurs des plateformes : la CJUE probablement saisie », Semaine Sociale Lamy, 4 février 2019, nº 1847.

21 CJUE, 20 déc. 2017, aff. C-434/15, paragraphe 38.

22 V. Roche, op.cit.

23 L. Gamet, op.cit.

24 L. Bento de Carvalho et S. Tourneaux, op.cit.

25 A. Fabre, "Le droit du travail peut-il répondre aux défis de l'ubérisation ? ", Revue du Droit du Travail, 2017, p.167.

26 V. Roche, op.cit. 
la Cour de cassation ${ }^{27}$, singulièrement au regard de tout dispositif semblant revêtir une portée disciplinaire ainsi que des modalités d'utilisation d'un système de géolocalisation. De surcroît, on soulignera que, si l'arrêt du 28 novembre 2018 garde le silence sur certains éléments susceptibles d'impacter le processus de requalification en relation salariée, ceux-ci devraient être pris en compte par toutes les plateformes désireuses d'échapper à un tel processus. Sont concernées la " propriété du matériel utilisé pour la réalisation de la prestation, [l'] influence des notes attribuées par les consommateurs au livreur, [l']intégration à un service organisé, [la] fixation du prix... ${ }^{28}$, sans parler d'une éventuelle fraude à la $\mathrm{loi}^{29}$.

Ce faisant, toutes les relations nouées avec des plateformes numériques ne devraient pas être systématiquement requalifiées, ce dont il ressort que la jurisprudence est loin d'être stabilisée, d'autant qu'appelées à se prononcer dans ce champ, les positions de la deuxième Chambre civile et de la Chambre criminelle sont encore inconnues. Pour l'heure, le fait est là : c'est une solution s'avérant tout à la fois classique et novatrice, rigoureuse et pragmatique, donc nuancée que la Chambre sociale a retenue; une solution problématique pour les opérateurs économiques mais que certains jugent "courageuse ", à l'heure où "le travail indépendant [semble s'imposer] comme modèle dominant de l'activité de plateforme de mise en relation par voie électronique .... ${{ }^{30}}^{30}$ une solution ainsi favorable aux travailleurs numériques mais dont la portée est incertaine. En effet, les démarches convergentes lancées par le législateur depuis 2016 ne risquent-elles pas d'en limiter la portée, voire de contourner cette analyse jurisprudentielle qui, d'ailleurs, s'inscrit dans un mouvement mondial de requalification ? ${ }^{31}$

\section{II - DEMAIN - VERS LA SUBSTITUTION D'UN STATUT LÉGAL DU TRAVAILLEUR NUMÉRIQUE À L'OFFICE DU JUGE ?}

À la question de savoir comment appréhender les relations tissées entre travailleurs et plateformes juridiques, c'est une réponse manifestement destinée à sécuriser celles-ci contre les risques d'une éventuelle requalification juridique que le législateur semble décidé à privilégier, comme en attestent les initiatives prises depuis 2016 (A) :

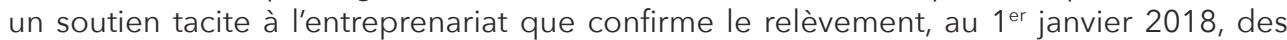
plafonds pour exercer comme micro-entrepreneur ${ }^{32}$ et qui peut être analysé comme " un signe implicite d'accompagnement du " désalariat " dans le contexte de l'émergence de l'économie collaborative $»^{33}$. Or, l'élaboration d'un tel statut légal n'aurait-elle pas pour conséquence de réduire la portée de la jurisprudence précédemment analysée et le rôle protecteur du juge ? Si la question peut se poser, la réponse est incertaine (B).

27 V. Roche, op.cit.

28 V. Roche, op.cit.

29 A. Jeammaud, «Uber, Deliveroo : requalification des contrats ou dénonciation d'une fraude à la loi ?", Semaine Sociale, 12 février 2018, nº 1802.

30 J-Ph. Lhernould, op.cit.

31 B. Gomez, «Take Eat Easy : une première requalification en faveur des travailleurs des plateformes », Semaine Sociale, 17 décembre 2018, nº 1841.

$32 \mathrm{CGl}$ art 50-0 (de 33200 euros à 70000 euros pour les prestations de service).

33 J-Ph. Lhernould, op.cit. 


\section{A - L'ÉDIFICATION D'UN STATUT DU TRAVAILLEUR NUMÉRIQUE : UN OBJECTIF RÉCURRENT DU LÉGISLATEUR}

C'est en 2016 que, sans se prononcer sur le statut juridique des travailleurs ubérisés et sans édicter de présomption de non-salariat, le législateur ${ }^{34} a$, pour la première fois, imposé quelques obligations aux plateformes numériques au nom de leur responsabilité sociale, via l'insertion, dans le Code du travail, de nouveaux droits et obligations imposés aux acteurs ${ }^{35}$. Côté droits, on trouve un droit d'accès à la formation professionnelle ainsi qu'un droit à la validation des acquis de l'expérience ${ }^{36}$, le coût de ces actions incombant à la plateforme dans des conditions fixées par décret ${ }^{37}$; on range également le droit de constituer une organisation syndicale et d'y adhérer ${ }^{38}$, ainsi que celui de déclencher des mouvements de refus concerté de fournir les services en vue de défendre des revendications professionnelles, sans qu'ils puissent être source de responsabilité contractuelle sauf abus, ni justifier une rupture des relations nouées avec la plateforme ${ }^{39}$. Côté obligations, on trouve notamment une obligation de prise en charge, par la plateforme, des cotisations dues au titre d'une assurance volontaire (ATMP), souscrite par le travailleur ${ }^{40}$.

Puis, plus récemment, c'est une seconde étape que la loi «Avenir professionnel ${ }^{41}$ devait franchir : une évolution freinée par le Conseil constitutionnel ${ }^{42}$, mais qui devrait revenir à l'ordre du jour à la faveur du projet de loi d'orientation des mobilités (projet de loi « LOM »), présenté en Conseil des ministres le 26 novembre 2018. Effectivement, son article 20 prévoit que toutes les plateformes pourraient établir une charte déterminant les conditions et modalités d'exercice de leur responsabilité sociale, définissant leurs droits et obligations ainsi que ceux des travailleurs avec lesquels elles sont en relation. Destinée à être publiée sur le site internet de la plateforme et à être annexée aux contrats ou aux conditions générales d'utilisation qui la lie aux travailleurs, cette charte - facultative $(\text { ? })^{43}$ et se voulant « éthique $»^{44}$ - devrait préciser notamment :

- les conditions d'exercice de l'activité professionnelle des travailleurs ayant recours à la plateforme, en particulier les règles selon lesquelles ils sont mis en relation avec les utilisateurs;

- les modalités visant à leur permettre d'obtenir un prix décent pour leur prestation ;

- les modalités de développement des compétences professionnelles et de sécurisation des parcours professionnels ;

34 Loi n 2016-1088 8 août 2016 relative au travail à la modernisation du dialogue social et à la sécurisation des parcours professionnels, JO, 9 août 2016, texte $n^{\circ} 3$.

35 Art L. 7341-1 à L. 7341-6. Décret n² 2017-774 du 4 mai 2017 relatif à la responsabilité sociale des plateformes de mise en relation par voie électronique

36 C. trav., art L 6111-1 et L 6411-1.

37 C. trav., art L. 7342-4.

38 C. trav., art. L. 7342-6.

39 C. trav., art. L. 7342-5.

40 C. trav., art. L. $7342-2$.

41 Loi n 2018-771, 5 sept. 2018, JO, 6 sept. 2018, texte $n^{\circ} 1$.

42 Cons. const., 4 sept. 2018, n 2018-769 DC, JurisData $n^{\circ}$ 2018-015955.

43 Sur cet aspect, voir G. Loiseau, «Travailleurs des plateformes numériques : le droit du travail en embuscade" "JCP, S, 2018, 1398.

44 M. Peyronnet, «Take Eat Easy contrôle et sanctionne des salariés», Revue de Droit du Travail, 2019, p.36. 
- les mesures visant à améliorer les conditions de travail et à prévenir les risques professionnels ;

- les modalités de partage d'informations et de dialogue entre la plateforme et les travailleurs sur les conditions d'exercice de leur activité professionnelle ;

- les modalités selon lesquelles les travailleurs sont informés de tout changement relatif aux conditions d'exercice de leur activité professionnelle ;

- la qualité de service attendue sur chaque plateforme et les circonstances pouvant conduire à une rupture des relations commerciales ainsi que les garanties prévues pour le travailleur dans ce cas;

- les garanties de protection sociale complémentaire négociées par la plateforme et dont les travailleurs peuvent bénéficier, notamment pour la couverture du risque décès, des risques portant atteinte à l'intégrité physique de la personne ou liés à la maternité, des risques d'incapacité de travail ou d'invalidité, des risques d'inaptitude, ainsi que la constitution d'avantages sous forme de pensions de retraite, d'indemnités ou de primes de départ en retraite ou de fin de carrière.

Et en contrepartie? Le texte prévoit que l'établissement d'une telle charte et le respect de ces engagements " ne [sauraient] caractériser l'existence d'un lien de subordination juridique entre la plateforme et les travailleurs». C'est à dire que « les conditions d'exercice de son activité par le travailleur ne pourraient pas constituer des indices de requalification en salariat dès lors, d'une part, que (de telles) conditions figurent dans la charte établie par la plateforme et, d'autre part, qu'elles peuvent être rattachées à l'un des thèmes énumérés par le texte de loi ...». «La charte protègerait donc, non seulement les travailleurs en leur offrant un statut social plus avantageux, mais également les plateformes contre le risque de requalification $\gg{ }^{45}$.

Dans ces conditions, et alors qu'un tel «projet s'inscrit dans la tendance actuelle visant à flexibiliser les relations de travail» ${ }^{46}$, est-ce à dire que le rôle du juge risque d'être anéanti car remplacé par ce statut légal des travailleurs ubérisés en cours d'édification? Rien n'est certain.

\section{B - L'ADOPTION D'UN STATUT LÉGAL DU TRAVAILLEUR NUMÉRIQUE : UNE ALTERNATIVE AU CONTRÔLE DU JUGE?}

S'il est vrai que l'instauration d'un statut légal des travailleurs numériques devrait exclure a priori toute intervention postérieure du juge, deux raisons laissent à penser que celui-ci pourrait poursuivre son office, au regret, certainement, des opérateurs numériques soucieux de sécurité juridique. Le fait est que, pour ceux-ci, une requalification judiciaire en travail salarié est lourde de conséquences puisque peuvent se juxtaposer : condamnation pour travail dissimulé visée par l'article. L. 8224-1 et suivants du Code du travail ; redressement opéré par l'Urssaf ; versement des sommes liées au contrat de travail (rappels de salaire, indemnité compensatrice de congés payés, primes...) ainsi qu'à sa rupture (indemnité compensatrice de préavis, indemnité de licenciement, indemnité forfaitaire pour travail dissimulé...).

C'est ainsi qu'un tel interventionnisme judicaire risque, en premier lieu, d'être rendu

45 V. Roche, op.cit.

46 B. Bossu, op.cit. 
possible par l'instauration d'une nouvelle présomption de non salariat dont la portée est cependant incertaine : en effet, "s'agira-t-il d'une présomption simple ou irréfragable ? Dans le premier cas, pourra-t-elle être renversée par la démonstration de l'existence d'un lien de subordination juridique "permanente", auquel cas il continuera d'être indispensable de rester attentif aux évolutions de l'interprétation prétorienne de cette notion ? ${ }^{47}$.

Quant à la seconde raison, elle est soulignée par Mr Loiseau ${ }^{48}$, selon qui la création d'une telle présomption " n'empêcherait pas un juge de retenir un tel lien au vu des circonstances de fait, dès l'instant où les éléments sur lesquels il se fonderait ne seraient pas ceux à propos desquels la plateforme a pris des engagements ": il s'agit là d'une capacité d'intervention qui pourrait être importante si, comme l'y poussent certains, le juge admettait une prise en compte de la dépendance économique dans l'établissement d'une relation subordonnée.

Voilà pourquoi sans doute, aiguillonné par le juge et tout en confirmant leur statut d'entrepreneur, le législateur est-il amené à renforcer les droits des travailleurs numériques garantis par les plateformes, afin de limiter d'autant les interstices dans lesquels le juge pourrait se glisser pour décider d'une éventuelle requalification de la relation contractuelle en relation salariée ${ }^{49}$. Ce faisant, c'est une "logique d'hybridation ${ }^{50}$ qui semble avoir la faveur des pouvoirs publics : une évolution clairement enclenchée mais dont l'issue reste incertaine pour deux raisons croisées. D'une part, elle «souligne le décalage entre deux dynamiques contraires (défense judiciaire du salariat/encadrement légal du non-salariat) [et auquel] il faudra s'attaquer pour parvenir à un statut complet et cohérent du travailleur de plateforme $»^{51}$ : un objectif dont la réalisation s'impose dans un contexte où le nombre de micro entrepreneurs augmente alors que leur statut favorise, pour beaucoup, une concurrence déloyale ${ }^{52}$. Mais, d'autre part, se pose la question de savoir jusqu'où aller dans ce processus de rapprochement des statuts, tant du point de vue du droit du travail que de celui de la protection sociale : un rapprochement dont une dernière illustration résulte de l'extension, à certaines conditions, de l'indemnisation chômage aux indépendants prévue par l'art 51 de la loi n 2018-771 du 5 septembre 2018. Certes, à ce rythme-là, peut-être les travailleurs des plateformes pourraient-ils, un jour, ne plus avoir « grand intérêt à demander la requalification en salariés $»^{53}$, du moins au regard de leur protection sociale : pour que cette évolution soit crédible, encore faut-il que les plateformes « versent leur écot à l'Etat providence $~^{54}$, alors même que leur « business model » repose sur l'objectif contraire de limitation des prélèvements sociaux ${ }^{55}$.

47 L. Bento de Carvalho et S. Tourneaux, op.cit.

48 G. Loiseau, op.cit.

49 G. Loiseau, op.cit.

50 J-Ph. Lhernould, op.cit.

$51 \mathrm{~J}$-Ph. Lhernould, op.cit.

52 « Micro entrepreneurs. Un succès en trompe-l'œil », Le Monde, 31 janvier 2019.

53 A. Taché (cité), "L'exécutif plaide pour une protection sociale des travailleurs 'ubérisés' », Le Monde, 10 mai 2018.

54 "L'exécutif plaide pour une protection sociale des travailleurs 'ubérisés' », Le Monde, 10 mai 2018.

55 O. Montel, «L'économie des plateformes : enjeux pour la croissance, le travail, l'emploi et les politiques publiques », Dares Analyses, 9 août 2017, n² 2017, p.213. 